\title{
A Novel Method for Preparation of Animal Models of Liver Damage: Liver Targeting of Carbon Tetrachloride in Rats
}

\author{
Takahiro Mukai, ${ }^{a}$ Kunihiro Mera, ${ }^{a}$ Koyo Nishida, ${ }^{a}$ Mikiro Nakashima, ${ }^{b}$ Hitoshi SASAKI,${ }^{b}$ \\ Toshiyuki SAKAEDA, ${ }^{c}$ and Junzo NAKAMURA ${ }^{*, a}$ \\ ${ }^{a}$ School of Pharmaceutical Sciences, Nagasaki University; 1-14 Bunkyo-machi, Nagasaki 852-8521, Japan: ${ }^{b}$ Department \\ of Hospital Pharmacy, Nagasaki University School of Medicine; 1-7-1 Sakamoto, Nagasaki 852-8501, Japan: and \\ ${ }^{c}$ Department of Hospital Pharmacy, School of Medicine, Kobe University; 7-5-2 Kusunoki-cho, Chuo-ku, Kobe 650-0017, \\ Japan. Received February 8, 2002; accepted August 23, 2002
}

\begin{abstract}
Animal models prepared by treatment with toxic compounds such as a carbon tetrachloride have been used to examine drug disposition in hepatic diseases. However, it is possible that these compounds accumulate and cause damage to other organs as they are administered systemically. In this study, we used the liver surface application technique to deliver a toxic compound to the liver to prepare an appropriate animal model in which only the liver is significantly damaged. To restrict the absorption area in the liver, a cylindrical diffusion cell was attached to the liver surface of male Wistar rats. Twenty-four hours after direct addition of carbon tetrachloride to the diffusion cell, plasma levels of glutamic-oxaloacetic transaminase (GOT) and glutamic-pyruvic transaminase (GPT), and hepatic malondialdehyde (MDA) concentration were increased, while there were no changes in plasma creatinine or renal MDA level. On the other hand, not only GOT, GPT and hepatic MDA, but also creatinine and renal MDA levels were markedly increased by p.o. and i.p. administration of carbon tetrachloride, suggesting renal damage. These results indicated that the animal models of liver damage prepared by utilizing drug delivery techniques to accumulate toxic compounds in the liver would enable us to investigate the precise effects of hepatic disorder on drug disposition.
\end{abstract}

Key words animal model; liver damage; carbon tetrachloride; liver targeting; rat

Drug disposition in various disease states is frequently different from that in the normal state. In particular, the liver plays an important role in drug metabolism and excretion in the body, and so there is increasing interest in investigation of pharmacokinetics in liver diseases. ${ }^{1,2)}$ To predict drug disposition in patients with liver diseases, animal models prepared by treatment with toxic compounds have been used. ${ }^{3-5}$ ) However, there is concern that toxic compounds might accumulate and cause toxic effects in other organs as they are administered systemically. There have been a few reports suggesting renal disorders in these animal models. ${ }^{6-8)}$ These findings raise the possibility that the pharmacokinetic results evaluated with these animal models might be affected by factors other than hepatic disorders. Therefore, an appropriate animal model in which only the liver is damaged is required to estimate the precise effects of hepatic disorder on drug disposition. Marked progress has been made in drug delivery techniques, ${ }^{9,10)}$ and their utilization for animal model preparation is expected to reduce damage to nontarget organs. However, to date there have been no reports of the application of such methods for this purpose.

In our laboratory, a liver surface application technique was developed for the purpose of drug delivery to the liver. ${ }^{11-17)}$ We established an experimental system utilizing a cylindrical diffusion cell attached to the rat liver surface to examine drug absorption from the liver surface without interference by absorption from other sites. The purpose of the present study was to prepare an appropriate animal model of liver damage with the use of this technique. We chose carbon tetrachloride $\left(\mathrm{CCl}_{4}\right)$ as a model toxic compound because of its wide use. After application of $\mathrm{CCl}_{4}$ to the rat liver surface, liver and kidney function were evaluated by monitoring several biochemical parameters. In addition, hepatic and renal lipid peroxidation levels induced by liver surface application of $\mathrm{CCl}_{4}$ were compared with those in rats treated orally or intraperitoneally.

\section{MATERIALS AND METHODS}

Materials $\mathrm{CCl}_{4}$ was obtained from Nacalai Tesque, Inc. (Kyoto, Japan). 2-Thiobarbituric acid (TBA) was obtained from Merck KGaA (Darmstadt, Germany). 1,1,3,3Tetraethoxypropane was purchased from Wako Pure Chemical Industries, Ltd. (Osaka, Japan). All other chemicals were of reagent grade.

Animals Male Wistar rats $(250-290 \mathrm{~g})$ were housed in cages in an air-conditioned room and maintained on a standard laboratory diet (MF, Oriental Yeast Co., Ltd., Tokyo, Japan) and water ad libitum. All experiments in the present study conformed to the Guidelines for Animal Experimentation in Nagasaki University.

Liver Surface Application (LSA) Experimental system for $\mathrm{CCl}_{4}$ application to rat liver surface is shown in Chart 1 . Rats were anesthetized with sodium pentobarbital $(50 \mathrm{mg} / \mathrm{kg}$,

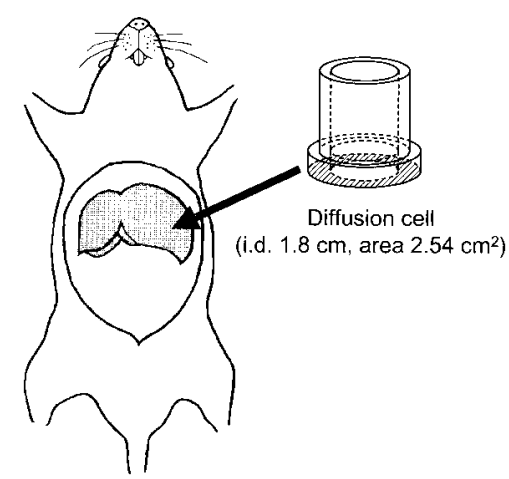

Chart 1. Experimental System for $\mathrm{CCl}_{4}$ Application to Rat Liver Surface 
i.p.) and a midline incision about $3 \mathrm{~cm}$ in length was made in the abdomen. A cylindrical glass diffusion cell (i.d. $1.8 \mathrm{~cm}$, area $2.54 \mathrm{~cm}^{2}$ ) was attached to the liver surface at the area of the left lateral lobe with a thin film of surgical adhesive (Aron Alpha, Sankyo Co., Ltd., Tokyo, Japan). One milliliter of $\mathrm{CCl}_{4}$ (LSA group) or saline (sham group) was added directly to the diffusion cell, and treatment was continued for 1 or $2 \mathrm{~h}$. The incision was sutured, and rats were housed in cages in an air-conditioned room with standard rat chow and water ad libitum. Twenty-four hours after administration of $\mathrm{CCl}_{4}$, rats were anesthetized with sodium pentobarbital and their body weights were measured. Blood was sampled from the femoral artery and plasma was separated by centrifugation at $15000 \mathrm{rpm}$ for $5 \mathrm{~min}$ (M-15-3; Sakuma, Tokyo, Japan). The liver and kidneys were perfused in situ with $50 \mathrm{ml}$ of saline, removed, and their wet weights were measured.

P.o. and i.p. Treatments Rats were anesthetized with sodium pentobarbital, and $\mathrm{CCl}_{4}$ in olive oil was administered orally or intraperitoneally at different doses. Twenty-four hours after administration of $\mathrm{CCl}_{4}$, samples of plasma, liver and kidney were prepared according to the procedure described above.

Assay of Plasma GOT, GPT, and Creatinine The plasma activities of glutamic-oxaloacetic transaminase (GOT) and glutamic-pyruvic transaminase (GPT) were determined with biochemical test kits (Wako Pure Chemical Industries, Ltd.) as indices of liver damage. As an index of kidney damage, the plasma concentration of creatinine was measured with a test kit (Wako Pure Chemical Industries, Ltd.).

Assay of Lipid Peroxidation in the Liver and Kidney As an index of lipid peroxidation, malondialdehyde (MDA) levels in the liver and kidney were determined according to the procedures of Uchiyama and Mihara. ${ }^{18)}$ Briefly, the organs were homogenized with cold $1.15 \% \mathrm{KCl}$ to make a $10 \%$ homogenate. To $0.5 \mathrm{ml}$ of $10 \%$ homogenate was added $3 \mathrm{ml}$ of $1 \%$ phosphoric acid and $1 \mathrm{ml}$ of $0.67 \%$ TBA solution. The mixture was heated for $45 \mathrm{~min}$ in a boiling water bath. After cooling, $4 \mathrm{ml}$ of $n$-butanol was added and mixed vigorously for $15 \mathrm{~min}$. The $n$-butanol phase was separated by centrifugation at $3500 \mathrm{rpm}$ for $30 \mathrm{~min}$ at $4{ }^{\circ} \mathrm{C}$ (RSL-05A; Sakuma). The MDA level was calculated from the difference in the absorbance at 535 and $520 \mathrm{~nm}$ using authentic MDA, prepared by hydrolysis of 1,1,3,3-tetraethoxypropane, as a standard.

Statistical Analysis Statistical analysis was performed by applying the unpaired Student's $t$-test, with $p<0.05$ considered statistically significant. All results are expressed as mean values \pm S.E. of at least three experiments.

\section{RESULTS}

Figure 1 shows GOT and GPT activities in plasma $24 \mathrm{~h}$ after administration of $\mathrm{CCl}_{4}$. LSA groups exhibited significantly higher GOT and GPT activities than sham groups. Furthermore, these activities increased with application time of $\mathrm{CCl}_{4}$. In p.o. and i.p. treatment groups, both GOT and GPT activities were significantly higher than those of normal rats. The enzyme activities of p.o. $100 \mu \mathrm{l}$ and i.p. $50 \mu \mathrm{l}$ groups were similar to those of the LSA $2 \mathrm{~h}$ group.

Creatinine concentrations in rat plasma at $24 \mathrm{~h}$ after administration of $\mathrm{CCl}_{4}$ are shown in Fig. 2. At each dose, p.o. and i.p. groups showed significantly higher creatinine levels

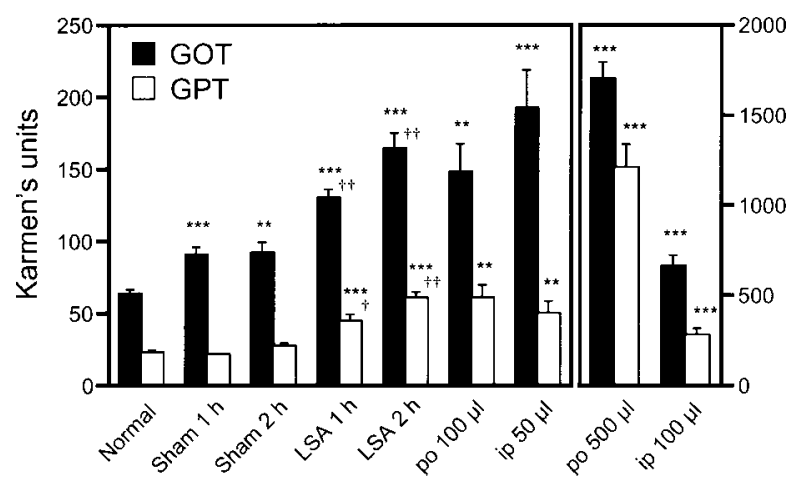

Fig. 1. Plasma GOT and GPT Activities $24 \mathrm{~h}$ after Administration of $\mathrm{CCl}_{4}$ to Rats under Several Conditions

Each bar represents the mean \pm S.E. of at least three experiments. Significant differences from the normal group $(* * p<0.01, * * * p<0.001)$ and from the sham group $(\dagger p<0.05, \dagger p<0.01)$.

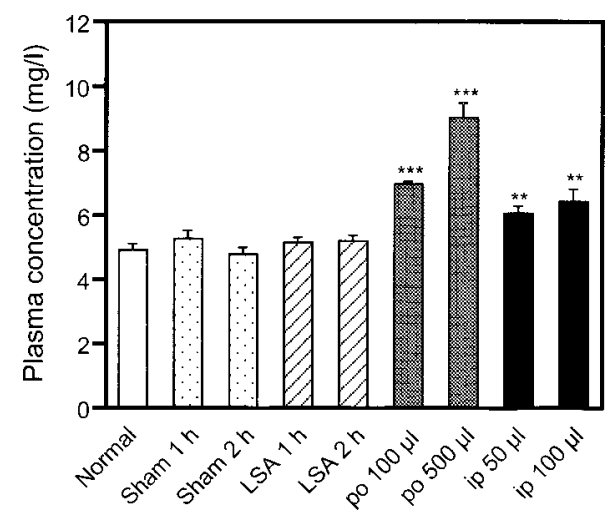

Fig. 2. Plasma Concentrations of Creatinine $24 \mathrm{~h}$ after Administration of $\mathrm{CCl}_{4}$ to Rats under Several Conditions

Each bar represents the mean \pm S.E. of at least four experiments. Significant differences from the normal group $(* * p<0.01, * * * p<0.001)$.

than the normal group. On the other hand, creatinine levels of LSA groups were not significantly different from those of sham and normal groups.

As an index of lipid peroxidation, MDA levels in the liver and kidney were determined at $24 \mathrm{~h}$ after administration of $\mathrm{CCl}_{4}$ (Figs. 3, 4). Since LSA $2 \mathrm{~h}$ group exhibited higher GOT and GPT activities and creatinine concentration than LSA $1 \mathrm{~h}$ group, the MDA levels of LSA $2 \mathrm{~h}$ group were evaluated. Although hepatic MDA level of LSA $2 \mathrm{~h}$ group was approximately twofold higher than that of the sham group (Fig. 3), the renal MDA level was not significantly increased (Fig. 4). In p.o. and i.p. groups, not only hepatic but also renal MDA levels were significantly higher than those of the normal group.

Liver weights (ratio of wet weights to the body weight) after administration of $\mathrm{CCl}_{4}$ are shown in Fig. 5. LSA groups exhibited significant increases in liver weight as compared to the sham groups. Similarly, liver weights of p.o. and i.p. groups were significantly greater than those of normal rats. Kidney weights after treatment with $\mathrm{CCl}_{4}$ (LSA, p.o. and i.p. groups) were not significantly different from those of sham and normal groups (data not shown).

\section{DISCUSSION}

$\mathrm{CCl}_{4}$-treated animals have been extensively used as experi- 


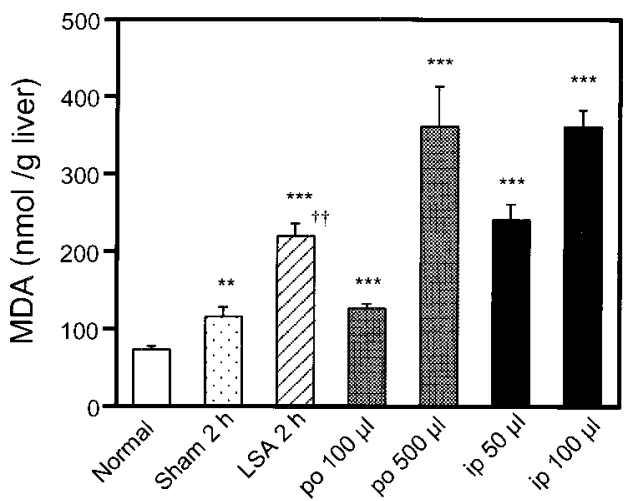

Fig. 3. Hepatic MDA Levels at $24 \mathrm{~h}$ after Administration of $\mathrm{CCl}_{4}$ to Rats under Several Conditions

Each bar represents the mean \pm S.E. of at least four experiments. Significant differences from the normal group $(* * p<0.01, * * * p<0.001)$ and from the sham group $(\dagger p<0.01)$.

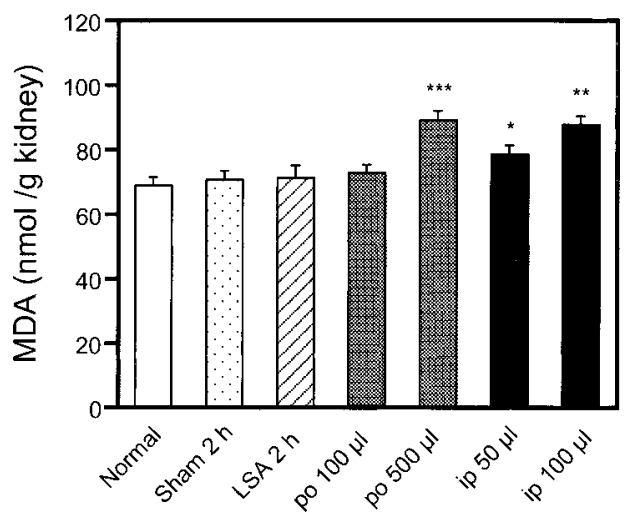

Fig. 4. Renal MDA Levels at $24 \mathrm{~h}$ after Administration of $\mathrm{CCl}_{4}$ to Rats under Several Conditions

Each bar represents the mean \pm S.E. of at least four experiments. Significant differences from the normal group $(* p<0.05, * * p<0.01, * * * p<0.001)$

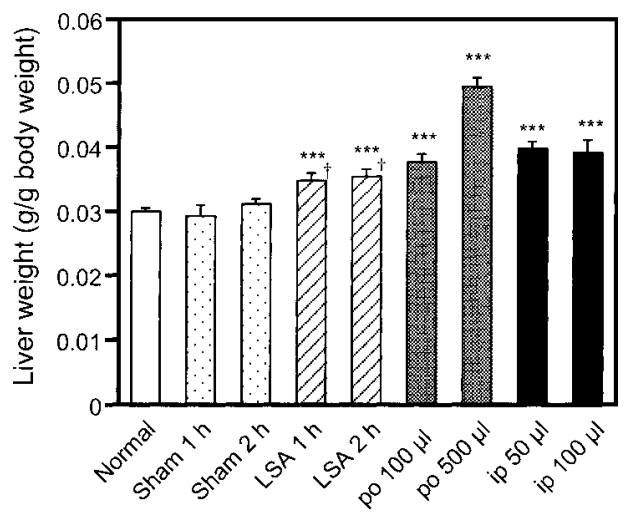

Fig. 5. Liver Weights (Ratio of Wet Weight to the Body Weight) at $24 \mathrm{~h}$ after Administration of $\mathrm{CCl}_{4}$ to Rats under Several Conditions

Each bar represents the mean \pm S.E. of at least four experiments. Significant differences from the normal group $(* * * p<0.001)$ and from the sham group $(\dagger p<0.05)$.

mental models of liver disease such as hepatic cirrhosis and drug-induced hepatopathy. ${ }^{19-22)}$ Numerous studies of the mechanisms operative in $\mathrm{CCl}_{4}$ hepatotoxicity demonstrated that the effect is dependent on the reduction to trichloromethyl radicals catalyzed by cytochrome P-450 (CYP) such as CYP2E1 and CYP2B in the endoplasmic reticulum. ${ }^{23-25)}$ The initial events of $\mathrm{CCl}_{4}$ metabolism evoke a cascade of secondary mechanisms that are responsible for ultimate plasma membrane disruption and cell death. ${ }^{26)}$

P.o. and i.p. administration of $\mathrm{CCl}_{4}$ caused increases not only in GOT and GPT but also in creatinine levels in the rat plasma (Figs. 1, 2). When $\mathrm{CCl}_{4}$ is administered systemically, the liver is commonly assumed to be the major target organ due to its high content of CYP. However, CYP2E1 is found in the rat kidney at a level of $5 \%$ of that found in the liver. ${ }^{27}$ ) Furthermore, Sanzgiri et al. reported that $\mathrm{CCl}_{4}$ administered systemically in rats was distributed in the kidney at higher concentrations than in the liver. ${ }^{28)}$ On the basis of these findings, renal damage caused by systemic administration of $\mathrm{CCl}_{4}$ is reasonable. Renal damage was supported by the renal elevation of lipid peroxidation levels (Fig. 4), led by production of trichloromethyl radicals. ${ }^{25,26,29)}$

To prepare an animal model in which only the liver is substantially damaged, liver targeting of $\mathrm{CCl}_{4}$ was attempted. Previously, we examined the absorption of various model drugs from the liver surface using an experimental system consisting of a cylindrical diffusion cell attached to the rat liver surface. Our results demonstrated that the tested drugs were adequately absorbed from the rat liver surface and efficiently accumulated in the liver. ${ }^{11-17)}$ Thus, we made use of this technique as a method of liver targeting of $\mathrm{CCl}_{4}$.

After application of $\mathrm{CCl}_{4}$ to the rat liver surface, plasma activities of GOT and GPT and hepatic lipid peroxidation levels were increased (Figs. 1, 3), suggesting hepatic damage. Application of $\mathrm{CCl}_{4}$ to the liver surface also produced a significant increase in liver weight (Fig. 5). This was characterized by centrilobular necrosis, ballooning degeneration, fatty deposition in the cytoplasm, and patchy infiltration of inflammatory cells. ${ }^{30,31)}$ Although plasma GOT and hepatic lipid peroxidation levels after application of saline to the rat liver surface (sham groups) were significantly higher than those of control (Figs. 1, 3), application of $\mathrm{CCl}_{4}$ induced significant increases in all parameters tested as compared to sham groups (Figs. 1, 3, 5). Thus, the hepatic damage by application of $\mathrm{CCl}_{4}$ to the rat liver surface was attributed not to the surgical procedure but to the toxicity of $\mathrm{CCl}_{4}$. On the other hand, plasma creatinine concentrations and renal lipid peroxidation levels after application of $\mathrm{CCl}_{4}$ to the rat liver surface were not significantly different from those in the sham and normal groups (Figs. 2, 4), suggesting that there was no renal damage. These findings indicated the selective accumulation of $\mathrm{CCl}_{4}$ in the liver by liver surface application, although the biodistribution of $\mathrm{CCl}_{4}$ was not be evaluated in this study.

In conclusion, the present study provided evidence that p.o. and i.p. administration of $\mathrm{CCl}_{4}$ impaired not only hepatic but also renal function. This finding suggested that animals treated systemically with $\mathrm{CCl}_{4}$ would be inadequate as models in which estimate the effects of hepatic disorder on drug disposition. The present study also indicated that application of $\mathrm{CCl}_{4}$ to the rat liver surface produced hepatic damage without impairing renal function. Currently, we are attempting to evaluate the drug metabolism and disposition in these animal models. Although additional studies are needed, the present findings suggest that it will be possible to investigate the precise effects of hepatic disorder on drug pharmacokinetics in animal models utilizing drug delivery techniques to accumulate toxic compounds in the liver. 
Acknowledgements We thank Kayoko Miyata, Rie Fujiwara, Tomomi Horimoto and Kazuko Kobayashi for skilled technical assistance. This work was supported in part by a Grant-in-Aid for Scientific Research from the Ministry of Education, Culture, Sports, Science and Technology, Japan.

\section{REFERENCES}

1) Koren G., Beatty K., Seto A., Einarson T. R., Lishner M., Ann. Pharmacother, 26, 363-371 (1992).

2) McLean A. J., Morgan D. J., Clin. Pharmacokinet., 21, 42-69 (1991).

3) Díaz-García J. M., Oliver-Botana J., Fos-Galve D., J. Pharm. Sci., 81, 768 - 772 (1992).

4) Kimura T., Nakayama S., Yamao T., Kurosaki Y., Nakayama T., Biol. Pharm. Bull., 16, 1140-1145 (1993).

5) Shibata N., Shimakawa H., Minouchi T., Yamaji A., Biol. Pharm. Bull., 16, 1130-1135 (1993).

6) Hirate J., Watanabe J., Ozeki, S., Chem. Pharm. Bull., 32, 2848-2850 (1984).

7) Li C., Nakayama S., Kurosaki Y., Nakayama T., Kimura T., J. Pharmacobio-Dyn., 13, 186-194 (1990).

8) Striker G. E., Smuckler E. A., Kohnen P. W., Nagle R. B., Am. J. Pathol., 53, 769-789 (1968).

9) Anderson J. H., Warren H. W., McArdle C. S., Clin. Pharmacokinet, 27, 191-201 (1994).

10) Torchilin V. P., Eur. J. Pharm. Sci., 11, S81-S91 (2000).

11) Nakamura J., Yoshida Y., Mera K., Mukai T., Nishida K., Sasaki H., Biol. Pharm. Bull., 22, 713-715 (1999).

12) Nishida K., Sato N., Sasaki H., Nakamura J., J. Pharm. Pharmacol., 46, 867-870 (1994).

13) Nishida K., Sato N., Sasaki H., Nakamura J., J. Pharm. Pharmacol., 47, 227-231 (1995).

14) Nishida K., Sato N., Sasaki H., Nakamura J., Biol. Pharm. Bull., 18,
$1548-1550$ (1995).

15) Nishida K., Sato N., Sasaki H., Nakamura J., J. Drug Target., 4, 141150 (1996).

16) Nishida K., Sato N., Nakakoga Y., Mukai T., Sasaki H., Nakamura J., J. Pharm. Pharmacol., 49, 976-980 (1997).

17) Nishida K., Nakakoga Y., Sato N., Kawakami S., Mukai T., Sasaki H., Sakaeda T., Nakamura J., Eur. J. Pharm. Biopharm., 50, 397-402 (2000).

18) Uchiyama M., Mihara M., Anal. Biochem., 86, 271-278 (1978).

19) Blondé-Cynober F., Plassart F., Rey C., Coudray-Lucas C., Moukarbel N., Poupon R., Giboudeau J., Cynober L., Ann. Nutr. Metab., 38, 238-248 (1994).

20) Pérez Tamayo R., Hepatology, 3, 112-120 (1983).

21) Reuber M. D., Glover E. L., J. Natl. Cancer Inst., 44, 419-427 (1970).

22) Rojkind M., Greenwel P., "Advances in Veterinary Science and Comparative Medicine," Vol. 37, ed. by Cornelius C. E., Academic Press, San Diego, 1993, pp. 333-355.

23) Kim S. G., Chung H. C., Cho J. Y., J. Pharmacol. Exp. Ther, 277, 1058-1066 (1996).

24) McCay P. B., Lai E. K., Poyer J. L., DuBose C. M., Janzen E. G., J. Biol. Chem., 259, 2135-2143 (1984).

25) McGregor D., Lang M., Mutat. Res., 366, 181-195 (1996).

26) Recknagel R. O., Glende E. A., Jr., Dolak J. A., Waller R. L., Pharmacol. Ther., 43, 139-154 (1989).

27) Ronis M. J. J., Huang J., Longo V., Tindberg N., Ingelman-Sundberg M., Badger T. M., Biochem. Pharmacol., 55, 123-129 (1998).

28) Sanzgiri U. Y., Srivatsan V., Muralidhara S., Dallas C. E., Bruckner J. V., Toxicol. Appl. Pharmacol., 143, 120-129 (1997).

29) Södergren E., Cederberg J., Vessby B., Basu S., Eur. J. Nutr., 40, 10 16 (2001).

30) Low D., Thomas N. W., Fry J. R., Toxicol. Lett., 81, 1-4 (1995).

31) Uemitsu N., Nakayoshi H., Toxicol. Appl. Pharmacol., 75, 1-7 (1984). 